\title{
ASSESSMENT OF CROPPING SYSTEM PRODUCTIVITY, PROFITABILITY AND ECONOMIC EFFICIENCY OF WHEAT
}

\author{
M. Nawaz ${ }^{1,2,}$, S, A. Anjum², U. Ashraf ${ }^{3}$, I. Khan $^{2}$, S. Hussain ${ }^{4}$, A. Zohaib ${ }^{2}$, Y. Hubiao ${ }^{5}$ and W. Zhiyong ${ }^{1, *}$ \\ ${ }^{1}$ Key Laboratory of Genetics and Germplasm Innovation of Tropical Special Forest Trees and Ornamental Plants, \\ Ministry of Education, College of Forestry, Hainan University, Haikou 570228, P. R. China; ${ }^{2}$ Department of Agronomy, \\ University of Agriculture, Faisalabad 38040, Pakistan \\ ${ }^{3}$ Department of Botany, University of Education, Lahore, Faisalabad-Campus, Faisalabad, 38000 Pakistan \\ ${ }^{4}$ State Key Laboratory of Rice Biology, China National Rice Research Institute, Hangzhou 310006, Zhejiang, PR China \\ ${ }^{5}$ Tropical Crops Genetic Resources Institute, Chinese Academy of Tropical Agricultural Sciences, Danzhou Hainan \\ 571737, China. \\ "Corresponding author’s e-mail: wangzhiyong@hainanu.edu.cn
}

\begin{abstract}
Cropping system throughout assessment exhibits importance for the crop production and cultural practices profitability. This field study was undertaken in semi-arid climate to devise suitable system. Wheat was sown in winter season while, summer crops include; cotton, maize and mungbean, which were sown under conventional tillage (CT), permanent beds (PB) and no tillage (NT) respectively. Yield and yield attributes were significantly influenced by tillage systems, highest productive tillers $\left(\mathrm{m}^{-2}\right)$, grains per spike and 1000-grain weight $(\mathrm{g})$ was observed in permanent beds $(\mathrm{PB})$. Grain yield $\left(\right.$ tha $^{-1}$ ) was also significantly higher $\left(4.03 \mathrm{tha}^{-1}\right)$ in PB. System productivity in terms of wheat equivalent yield (WEY) of maize-wheat (MW) under PB was higher $\left(10.26 \mathrm{t} \mathrm{ha}^{-1}\right)$ followed by mungbean-wheat in permanent bed $\left(9.40 \mathrm{t} \mathrm{ha}^{-1}\right)$ and cotton-wheat in conventional tillage $\left(9.26\right.$ tha $\left.^{-1}\right)$. Highest cultivation cost was observed in the cotton-wheat followed by maize-wheat under conventional tillage and least was observed in the mungbean-wheat in no tillage. Cotton-wheat (CW) gave maximum net field benefit (US\$. 1606.53ha ${ }^{-1}$ ) in under PB followed by mungbean-wheat (MbW) (US\$.1411.72ha ${ }^{-1}$ ). However, maximum benefit cost ratio (BCR) (3.77) was recorded in mungbean-wheat system. Moreover, the cotton-wheat in permanent bed system was the best possible option for acquiring high production and land use efficiency.
\end{abstract}

Keywords: Cropping system; wheat; net return; profitability; system productivity.

https://doi.org/10.36899/JAPS.2020.2.0041

Published online March 02, 2020

\section{INTRODUCTION}

World population is supposed to increase the figure of 9000' million by the year 2050 (FAO 2014). The challenge to feed this gigantic population is not easy task. Wheat known to be an essential food of human beings and is most widely grown crop (Dawe 2008). Above twenty percent calories to human provided by wheat worldwide (FAO 2014; Peter 2011; Braun et al., 2010). The average grain yield is much lower in Pakistan, regardless of higher yield potential (USDA 2016). Two principal constraints responsible for the lowering the crop yield includes; dependence on conventional practices and unwise selection of crop without assessing the capacity of the soil for crop production (Shehzad et al. 2016).

Monoculture (cereal-cereal) system becomes increasingly prone to insect pests and diseases attack owing to less diversity in crop selection (Ratnadass et al. 2012; David 2002). Thus, agricultural system productivity is decreasing constantly under such systems (Tilman et al. 2002). The recent persistence of growers on conventional tillage (CT) practices for making an ideal seed-bed resulted in hard-pan in root zone, which adversely affects crop productivity by hindering the growth of plant root system (Zhang et al. 2006; Micucci and Taboada 2006; Bertolino et al. 2010; Shahzad et al. 2016), eventually loss in terms of profitability encored at farm level.

Cereal based cropping system exhibits great importance in food chain/security (Timsina and Connor 2001; Ladha et al. 2003). Tanaka et al. (2002) stated rotations as different crops were grown in a specific order and/or specified pattern though, crop multifariousness was quite limited over the area. Higgs et al. (1990) and Crookston (1995) reported that the differences in crop yield were related with crop rotation was implied to the rotation effect. The meliorative effect of inclusion of legumes in cereal-based cropping system has long been documented (Bari and Islam 2009).

Conservation tillage system have advantage of increased yield of crops (wheat and cotton) in permanent beds compared with conventional system under irrigated semiarid climate (Boulal et al. 2012; Naresh et al. 2012). Moreover, Kienzler et al. (2012) reported that yield under 
conservation tillage practices were less and inconsistent in irrigated regions. In cotton-wheat cropping system, seeding of wheat after cotton resulted in incorporation of organic matter in soil in the form of cotton leaves and weed residues (Khan and Khaliq 2005). Zandastra (1976) revealed cropping system as crop production enterprise used to drive benefits from a given resource and specific environment condition. Conservation tillageis advantageous to soil biological, physical environment (Gathala et al. 2011; Sainju et al. 2008), and chemical properties by minimal soil disturbance and implementation of varied crop sequences (Farooq et al. 2011; Friedrich et al. 2017) resulting in augmentation of crop productivity. NT reduce cost, save soil moisture and increased yield (De Vita et al. 2007; Erenstein et al. 2008), reduced energy consumption (Garcua-Torres 2000), thus become more attractive than CT among the farmers (Hobbs, 2007).

Though number of studies was executed to evaluate of the impact of crop sequence on the wheat performance under various tillage practices, but there was no enlightenment on the economics of wheat based system in relation to different tillage regimes. Therefore, this field experiment was aimed at assessing the economic efficiency of various wheat-based cropping systems under different tillage practices.

\section{MATERIALS AND METHODS}

Site, soil and climatic conditions: A two-year field experiment was conducted at Agronomic Research Area, University of Agriculture, Faisalabad (longitude $73^{\circ}-76^{\circ}$ E, $31^{\circ}-26^{\circ} \mathrm{N}$ and $135 \mathrm{~m}$ elevation) Pakistan during 2014-15. The soil at Faisalabad was a sandy loam from the Lyallpur series. According to chemical analysis, soil had $\mathrm{pH} 7.6,0.38 \%$ soil organic matter, $0.35 \mathrm{dS} \mathrm{m}^{-1}$ electrical conductivity, $0.05 \%$ total $\mathrm{N}, 6.88 \mathrm{mg}$ extractable $\mathrm{P} \mathrm{kg}{ }^{-1}, 163 \mathrm{mg}$ extractable $\mathrm{K} \mathrm{kg}^{-1}$.

Experimental details: The economics and profitability of wheat sown under tillage systems was assessed in four different wheat-based cropping systems. The cropping systems included in the experiment were 1) cotton-wheat, 2) maize-wheat, 3) mungbean-wheat system. The tillage practices evaluated were 1) conventional tillage (CT), 2) permanent bed, 3) (PB) no tillage (NT). The experiment was laid out according to randomized complete block design with split plot arrangements. Tillage practices were assigned to main plots, while cropping systems were arranged in sub-plots having three replications. The size of main and sub-plots was $20 \mathrm{~m} \times 15 \mathrm{~m}$, and $5 \mathrm{~m} \times 3$ $\mathrm{m}$, respectively.

Crop husbandry: Before seedbed preparation, presoaking irrigation of four inch was applied before cultivation. When soil reached at field capacity, seedbeds were prepared as per treatment. All the crops included in the study were sown according to recommendations by the Department of Agriculture Extension, Punjab, Pakistan (Shahzad et al. 2017). In total, five irrigations were applied to wheat crop in order to avoid the negative effects of moisture stress throughout the crop season (Table 1). All the crops were harvested manually at their harvest maturity. At harvest maturity, three central rows from each plot of a given crop were harvested, sun-dried for one week, threshed manually, grains were separated, and weighed to calculate grain yield which was converted into tons per hectare. The grain yields of all crops were adjusted at $10 \%$ grain moisture contents. Data regarding grain yield of wheat crop used to compute the economics. The yield data of the crops was recorded for the computation of the economics and profitability.

Expenses incurred: The variable and fixed costs incurred were calculated according to current market rates of inputs in US Dollar (US\$). For summer crops, the fixed cost included the cost of fertilizers transport/application and the land rent. The cost of land preparation, seed, sowing, irrigation application, fertilizers, and harvesting varied among all summer crops. Similarly, for wheat crop, the total fixed cost included seed cost, sowing, fertilizers transportation, irrigation and fertilizers application, and crop harvesting. The cost of land preparation was considered as variable cost. Total expenditure incurred was computed by adding the variable and fixed costs of summer and winter crops for respective treatments.

Economic and marginal analysis: The economic and marginal analysis was performed following the CIMMYT (1988). The grain and straw yields from each crop were multiplied with the unit market price to determine the income (US\$ ha ${ }^{-1}$ ). The market rates of product in US dollar (US\$) per 40 kilograms (kg) are as follow; Cotton $=$ US\$ 25 per $40 \mathrm{~kg}$; Maize= US\$ 9 per $40 \mathrm{~kg}$; Mungbean $=$ US\$36 per $40 \mathrm{~kg}$. Total income of a cropping system was calculated by adding the income of grain and straw yields of each summer and winter crop. Net benefit returns were obtained by subtracting total expenditures from the relevant total income. Benefit-cost ratio (BCR) was calculated by dividing the total income by total expenditures. Moreover, marginal rate of return (MRR) was also calculated to find out the most cost-effective treatment of the study. MRR was determined by arranging the variable cost of treatments in descending order. The respective net field benefits of treatments were also placed in order. Changes in variable cost and net field benefits were calculated by subtraction. Data were analyzed for partial budget analysis and marginal analysis. The performance of different cropping system in terms of wheat equivalent yield (WEY) was computed using the formula: $W E Y($ crop $)=Y_{a}\left(P_{x}\right) / P_{b}$.

Where, $Y_{a}$ is the yield of crop ' $a$ ' ( $t /$ ha of economic harvest), $\mathrm{P}_{\mathrm{a}}$ is the price of crop ' $\mathrm{a}$ ', and $\mathrm{P}_{\mathrm{b}}$ is 
the price of wheat (Schlegel et al. 2016). Production efficiency in terms of $\mathrm{kg} / \mathrm{ha} /$ day was calculated according to (Kundu and Mahapatra 2014).

Statistical analysis: Analysis of variance (ANOVA) test was performed using statistical software 'Statistix 8' (Analytical software, Tallahassee, Florida, USA) for windows, while difference among the treatment means was determined using Tukey's (HSD) at $\mathrm{P} \leq 0.05$ and $\mathrm{P} \leq$ 0.01 .

\section{RESULTS AND DISCUSSION}

Production in various cropping/tillage systems: Tillage significantly affected the yield and yield parameter of wheat, as wheat yield under permanent beds was significantly $(\mathrm{P} \leq 0.05)$ higher by $10.11 \%$ compared to conventional tillage planting system (Table 2). However, wheat planted in no tillage yielded 21.04\% lower compared to conventional tillage. The yield of wheat also differed significantly $(\mathrm{P} \leq 0.05)$ under different cropping systems. Maximum wheat grain yield (3.60 tha $\left.{ }^{-1}\right)$ was recorded under maize-wheat system followed by cottonwheat (3.53 tha $\left.^{-1}\right)$ as compared to other systems. On other hand, permanent beds gave highest yield (4.03 t $\mathrm{ha}^{-1}$ ) than other tillage systems. This increased wheat production in bed sowing ascribed to the provision of improved water and nutrient uptake and soil health to the crop (Shahzad et al. 2016; Khan et al. 2012). The number of tillers $\mathrm{ha}^{-1}$, number of grains spike ${ }^{-1}$ and 1000 -grain weight were significantly affected by diversified tillage treatments (Table 2).

Income beared from various cropping/tillage systems: Different cropping sequences were economically analyzed (Table 3), revealed that cotton-wheat system required highest cost of cultivation in conventional tillage. The greatest cost was incurred due to the more investment in terms of management practices (tillage operations) and fertilization. The lowest cost of cultivation was incurred in mungbean-wheat cultivated under no tillage. Permanent bed saves capital, requires less tillage operation and higher benefit: cost ratio (BCR) compared to the conventional tillage Shah et al. (2016). Gross and net income was also highest for cotton-wheat system in permanent bed.

Farmers are interested in high benefits from the crops therefore; net field benefits (NFB) were calculated against the variable costs. Maximum net field benefit (US\$. 948.67ha ${ }^{-1}$ ) was observed in cotton followed by mungbean (US\$.733.72 $\mathrm{ha}^{-1}$ ) and maize (US\$. 425.40ha ${ }^{-1}$ ) in permanent bed during summer season (Table $4 \mathrm{a}$ ). While, minimum net field benefit was found in the no tillage. Table $4 \mathrm{~b}$ reveals that maximum net field benefit (US\$. 664.11 ha ${ }^{-1}$ ) was observed in wheat grown after maize in permanent bed followed by the cotton-wheat (US\$. $657.86 \mathrm{ha}^{-1}$ ) in conventional tillage. The minimum net field benefit (US\$. 428.47 $\mathrm{ha}^{-1}$ ) was observed in the wheat grown after mungbean in no tillage system. Thus, this study proved no tillage as low-input tool for cultivation compared to other tillage systems on account of no land preparation and minimal fuel consumption (Shahzad et al. 2017; Akbarnia and Farhani 2014; Iqbal et al. 2002).

Benefit cost ratio (BCR) is also important indicator to farmers because they are interested in the increased net returns by managing the total cost of production. In summer, maximum BCR (2.42) was found in the mungbean under permanent bed followed by mungbean (2.27) in conventional tillage while, lowest BCR (1.21) was observed in no tillage cultivated maize (Table 4a). During the winter season, maize-wheat system in permanent bed gave highest (2.78) BCR followed by the cotton-wheat in permanent bed (2.76) and least BCR (2.22) was observed in mungbean-wheat in no- tillage (Table $4 \mathrm{~b}$ ). The income gained from bed sown wheat in terms of net economic returns and BCR were also highest in rice-wheat cropping system (Shahzad et al. 2017; Conner et al. 2003). Maximum BCR in maize-wheat system in permanent bed was attributed to its less cost of production and more gross income as compare to other treatments. Marginal analysis also suggested more benefits of bed sowing in this experiment. Likewise, highest marginal rate of return was observed in maize, followed by cotton and then mungbean under permanent bed respectively, during summer 2014 (Table 5a). In winter 2014-15 highest marginal rate of return was obtained in cotton-wheat followed by the maize-wheat rotation followed by mungbean-wheat system in permanent bed respectively (Table 4a).

Land resource use efficiency (LRUE) and production efficiency (PE) was conferred in Table 6. Highest land resource use efficiency (87.12\%) was recorded in the cotton-wheat sequence. Moreover, highest production efficiency $\left(23.07 \mathrm{kgha}^{-1} \mathrm{day}^{-1}\right)$ was also recorded in cotton-wheat under permanent bed system; Khalid et al. (2014) also found the same results in the cotton-wheat system. The maize-wheat system recorded as second highest efficient system of production (20.06 $\mathrm{kgha}^{-1} \mathrm{day}^{-1}$ ) with land resource use efficiency of 70.96 percent. The lowest production efficiency (13.24 kgha ${ }^{1}$ day $^{-1}$ ) was recorded in no tillage cotton-wheat system; while, lowest land resource use efficiency was observed in the maize-wheat system under all the tested tillage treatment. The mungbean-wheat cropping system utilized land for shorter duration thus had lowest land resource use efficiency among all the tested treatments 
Table 1. Agronomic practices of individual crop followed during experimentation.

\begin{tabular}{lcccccc}
\hline Crop & Variety & $\begin{array}{c}\text { Seed rate } \\
\left(\mathbf{k g h a}^{-1}\right)\end{array}$ & $\begin{array}{c}\text { Spacing } \\
(\mathbf{c m} \times \mathbf{c m})\end{array}$ & $\begin{array}{c}\text { No. of } \\
\text { irrigation }\end{array}$ & $\begin{array}{c}\text { Fertilizers N:P:K } \\
\left(\mathbf{k g h a}^{-1} \mathbf{)}\right.\end{array}$ & $\begin{array}{c}\text { Intercultural } \\
\text { operation }^{-}\end{array}$ \\
\hline Wheat & FSD-2008 & 120 & 22.5 & 5 & $120: 80: 0$ & 2 \\
Cotton & MNH-886 & 25 & $75 \times 75$ & 12 & $200: 115: 0$ & 3 \\
Maize & DK-6789 & 25 & $75 \times 75$ & 8 & $250: 115: 0$ & 2 \\
Mungbean & Mung-6 & 25 & $8 \times 30$ & 3 & $23: 57: 0$ & 1 \\
\hline
\end{tabular}

Table 2. Effects of tillage and crop rotation on yield and yield attributes of wheat.

\begin{tabular}{|c|c|c|c|c|c|}
\hline $\begin{array}{c}\text { Main effect/ } \\
\text { level }\end{array}$ & $\begin{array}{l}\text { Productive } \\
\text { tillers }\left(\mathbf{m}^{-2}\right)\end{array}$ & Grains per spike & $\begin{array}{l}\text { 1000-grain } \\
\text { weight (g) }\end{array}$ & $\begin{array}{c}\text { Grain yield } \\
\left(\mathrm{t} \mathrm{ha}^{-1}\right)\end{array}$ & $\begin{array}{l}\text { Biological yield } \\
\left(\mathrm{t} \mathrm{ha}^{-1}\right)\end{array}$ \\
\hline \multicolumn{6}{|l|}{ Tillage } \\
\hline Conventional & $232 \pm 2.96 \mathrm{a}$ & $42 \pm 0.67 \mathrm{a}$ & $41.34 \pm 0.32 \mathrm{a}$ & $3.66 \pm 0.14 b$ & $10.24 \pm 0.14 \mathrm{~b}$ \\
\hline Permanrnt bed & $242 \pm 3.18 \mathrm{a}$ & $44 \pm 0.12 \mathrm{a}$ & $42.53 \pm 0.15 \mathrm{a}$ & $4.03 \pm 0.22 \mathrm{a}$ & $10.65 \pm 0.12 \mathrm{a}$ \\
\hline No till & $199 \pm 2.60 \mathrm{~b}$ & $39 \pm 0.30 \mathrm{~b}$ & $39.13 \pm 0.23 b$ & $2.89 \pm 0.11 \mathrm{c}$ & $8.33 \pm 0.11 \mathrm{c}$ \\
\hline \multicolumn{6}{|l|}{ Rotation } \\
\hline Cotton-wheat & $226 \pm 1.76$ & $39 \pm 0.14$ & $40.88 \pm 0.35$ & $3.53 \pm 0.14 \mathrm{ab}$ & $9.73 \pm 0.10$ \\
\hline Maize-wheat & $225 \pm 2.54$ & $39 \pm 0.20$ & $41.26 \pm 0.24$ & $3.60 \pm 0.17 \mathrm{a}$ & $9.76 \pm 0.05$ \\
\hline Mung-wheat & $222 \pm 1.64$ & $39 \pm 0.34$ & $40.88 \pm 0.33$ & $3.45 \pm 0.19 b$ & $9.73 \pm 0.07$ \\
\hline Tillage & & & & & \\
\hline Crop & NS & NS & NS & "** & NS \\
\hline Tillage $\mathrm{x}$ crops & NS & NS & NS & ${ }^{* *}$ & \\
\hline
\end{tabular}

Means values of three replicates \pm SE. Mean values followed by the similar letters within columns don't differ significantly $(\mathrm{P} \leq 0.05)$. One $\operatorname{star}\left({ }^{*}\right)$ mean values show significant, two stars $\left(^{* *}\right)$ show highly significant mean values.

Table 3. Economic analysis of wheat under different wheat based cropping system.

\begin{tabular}{|c|c|c|c|c|c|}
\hline Tillage & Cropping system & $\begin{array}{c}\text { a Cost of cultivation } \\
\left(\text { US\$. } \text { ha }^{-1}\right)\end{array}$ & $\begin{array}{c}{ }^{b} \text { Gross Return } \\
\text { (US\$. ha }{ }^{-1} \text { ) }\end{array}$ & $\begin{array}{l}\text { cet Return } \\
\left(\text { US\$. ha }{ }^{-1}\right)\end{array}$ & $\begin{array}{c}{ }^{\mathrm{d}} \text { Return per } \\
\text { invested } \\
(\mathrm{US} \$)\end{array}$ \\
\hline \multirow{3}{*}{ Conventional } & Cotton-wheat & $1172.46 \pm 1.87 \mathrm{a}$ & $2072.43 \pm 1.72 b$ & $899.98 \pm 1.59 c$ & $2.31 \pm 0.05 \mathrm{c}$ \\
\hline & Maize-wheat & $1083.53 \pm 0.67 b$ & $1792.89 \pm 1.02 \mathrm{c}$ & $709.36 \pm 0.93 \mathrm{e}$ & $2.53 \pm 0.01 b$ \\
\hline & Mungbean-wheat & $700.92 \pm 1.72 \mathrm{~g}$ & $1571.40 \pm 2.69 \mathrm{e}$ & $870.49 \pm 1.52 \mathrm{~cd}$ & $1.81 \pm 0.06 \mathrm{~d}$ \\
\hline \multirow[t]{3}{*}{ Permanent bed } & Cotton-wheat & $1073.81 \pm 1.97 \mathrm{c}$ & $2286.96 \pm 1.29 \mathrm{a}$ & $1213.16 \pm 1.03 \mathrm{a}$ & $1.89 \pm 0.05 \mathrm{~d}$ \\
\hline & Maize-wheat & $988.39 \pm 0.75 \mathrm{e}$ & $1800.29 \pm 1.09 c$ & $811.90 \pm 1.02 \mathrm{~d}$ & $2.22 \pm 0.01 \mathrm{c}$ \\
\hline & Mungbean-wheat & $666.74 \pm 0.64 \mathrm{~h}$ & $1698.61 \pm 0.67 d$ & $1031.87 \pm 0.59 b$ & $1.65 \pm 0.02 \mathrm{e}$ \\
\hline \multirow[t]{3}{*}{ No tillage } & Cotton-wheat & $995.37 \pm 1.99 \mathrm{~d}$ & $1596.45 \pm 1.25 \mathrm{e}$ & $601.08 \pm 0.98 f$ & $2.66 \pm 0.0 \mathrm{~b}$ \\
\hline & Maize-wheat & $934.23 \pm 1.58 \mathrm{f}$ & $1401.30 \pm 1.46 \mathrm{f}$ & $467.07 \pm 1.23 \mathrm{~g}$ & $3.00 \pm 0.01 \mathrm{a}$ \\
\hline & Mungbean-wheat & $575.54 \pm 1.09 \mathrm{i}$ & $1226.59 \pm 1.09 \mathrm{~g}$ & $651.05 \pm 0.98 \mathrm{ef}$ & $1.88 \pm 0.01 \mathrm{~d}$ \\
\hline
\end{tabular}

${ }^{\mathrm{a}}$ Cost of cultivation; total cost form sowing to harvesting, ${ }^{\mathrm{b}}$ Gross Return; income without excluding the cost, ${ }^{\mathrm{c}}$ Net Return; income excluding the cost, ${ }^{\mathrm{d}}$ Return per invested; income by investing 1US\$. 
Table 4a. Effect of different tillage system on net field benefit and BCR of Kharif crops.

\begin{tabular}{|c|c|c|c|c|c|c|c|c|}
\hline & Treatment & $\begin{array}{l}\text { Yield } \\
\left(\mathrm{t} \mathrm{ha}^{-1}\right)\end{array}$ & $\begin{array}{l}\text { Yield Value } \\
\text { (US\$. } \text { ha }^{-1} \text { ) }\end{array}$ & $\begin{array}{c}\text { Straw Value } \\
\left(\text { USS. ha } \mathbf{h a}^{-1}\right)\end{array}$ & $\begin{array}{l}{ }^{\text {a }} \text { Gross income } \\
(\text { US\$. ha }\end{array}$ & $\begin{array}{l}\text { Total cost } \\
\left(\text { US\$. } \text { ha }^{-1}\right)\end{array}$ & $\begin{array}{l}{ }^{\text {b }} \text { Net field } \\
\text { benefit } \\
\left.\text { (USS. ha }{ }^{-1}\right)\end{array}$ & BCR \\
\hline \multirow{3}{*}{ Cotton } & Traditional tillage & $2.91 \pm 0.03^{b}$ & $1724.26 \pm 6.40^{b}$ & $94.95 \pm 0.0$ & $1819.22 \pm 3.94^{\mathrm{b}}$ & $1144.75 \pm 1.30^{\mathrm{a}}$ & $674.47 \pm 4.61^{b}$ & $1.59 \pm 0.02^{\mathrm{b}}$ \\
\hline & Permanent bed & $3.21 \pm 0.02^{\mathrm{a}}$ & $1907.91 \pm 4.08^{\mathrm{a}}$ & $94.95 \pm 0.0$ & $2002.86 \pm 5.8^{\mathrm{a}}$ & $1054.19 \pm 0.69^{b}$ & $948.67 \pm 5.01^{\mathrm{a}}$ & $1.90 \pm 0.01^{\mathrm{a}}$ \\
\hline & No tillage & $2.19 \pm 0.05^{\mathrm{c}}$ & $1299.62 \pm 3.40^{\mathrm{c}}$ & $94.95 \pm 0.0$ & $1394.57 \pm 3.90^{\mathrm{c}}$ & $1007.32 \pm 1.82^{\mathrm{c}}$ & $387.25 \pm 4.52^{\mathrm{c}}$ & $1.38 \pm 0.03^{\mathrm{c}}$ \\
\hline \multirow{3}{*}{ Maize } & Traditional tillage & $6.38 \pm 0.04^{\mathrm{a}}$ & $1362.90 \pm 7.89^{\mathrm{a}}$ & $63.18 \pm 0.01$ & $1426.08 \pm 7.34^{\mathrm{a}}$ & $1024.57 \pm 0.51^{\mathrm{a}}$ & $401.51 \pm 6.84^{\mathrm{a}}$ & $1.39 \pm 0.01^{\mathrm{b}}$ \\
\hline & Permanent bed & $6.12 \pm 0.04^{\mathrm{b}}$ & $1307.49 \pm 8.27^{\mathrm{b}}$ & $58.81 \pm 0.04$ & $1366.30 \pm 4.22^{\mathrm{b}}$ & $940.89 \pm 0.52^{\mathrm{b}}$ & $425.40 \pm 3.74^{\mathrm{a}}$ & $1.45 \pm 0.01^{\mathrm{a}}$ \\
\hline & No tillage & $4.96 \pm 0.02^{\mathrm{c}}$ & $1060.53 \pm 3.30^{\mathrm{c}}$ & $56.69 \pm 0.03$ & $1117.22 \pm 5.74^{\mathrm{c}}$ & $924.17 \pm 0.21^{\mathrm{c}}$ & $193.05 \pm 5.54^{\mathrm{b}}$ & $1.21 \pm 0.01^{\mathrm{c}}$ \\
\hline \multirow{3}{*}{ Mungbean } & Traditional tillage & $1.35 \pm 0.01^{\mathrm{b}}$ & $1154.15 \pm 6.68^{\mathrm{b}}$ & $28.49 \pm 0.0$ & $1182.63 \pm 6.68^{\mathrm{b}}$ & $520.71 \pm 0.67^{\mathrm{a}}$ & $661.92 \pm 6.01^{b}$ & $2.27 \pm 0.01^{\mathrm{b}}$ \\
\hline & Permanent bed & $1.43 \pm 0.02^{\mathrm{a}}$ & $1220.89 \pm 6.94^{\mathrm{a}}$ & $28.49 \pm 0.0$ & $1249.38 \pm 4.69^{\mathrm{a}}$ & $515.66 \pm 1.69^{\mathrm{b}}$ & $733.72 \pm 5.24^{\mathrm{a}}$ & $2.42 \pm 0.03^{\mathrm{a}}$ \\
\hline & No tillage & $1.00 \pm 0.02^{\mathrm{c}}$ & $856.01 \pm 4.16^{\mathrm{c}}$ & $28.49 \pm 0.0$ & $884.50 \pm 3.66^{\mathrm{c}}$ & $447.51 \pm 1.42^{\mathrm{c}}$ & $436.99 \pm 2.75^{\mathrm{c}}$ & $1.98 \pm 0.03^{\mathrm{c}}$ \\
\hline
\end{tabular}

Table 4b. Effect of different tillage system and crop rotation on total cost, net field benefit and benefit cost ratio.

\begin{tabular}{|c|c|c|c|c|c|c|c|c|c|}
\hline Tillage & $\begin{array}{l}\text { Cropping } \\
\text { system }\end{array}$ & $\begin{array}{l}\text { Wheat Yield } \\
\quad\left(\mathrm{t} \mathrm{ha}^{-1}\right)\end{array}$ & $\begin{array}{c}\text { Wheat Yield } \\
\text { Value } \\
\left(\text { US\$. ha }^{-1}\right)\end{array}$ & $\begin{array}{l}\text { Straw yield } \\
\left(\mathrm{t} \mathrm{ha}^{-1}\right)\end{array}$ & $\begin{array}{c}\text { Straw Value } \\
\left(\text { US\$. } \text { ha }^{-1}\right)\end{array}$ & $\begin{array}{c}{ }^{\mathrm{a}} \text { Gross } \\
\text { income } \\
\left(\mathrm{US \$} \$ \mathrm{ha}^{-1}\right)\end{array}$ & $\begin{array}{c}\text { Total cost } \\
\left(\text { US\$. ha }^{-1}\right)\end{array}$ & $\begin{array}{l}{ }^{\text {b }} \text { Net field } \\
\text { benefit } \\
\left(\text { US\$. ha }{ }^{-1}\right)\end{array}$ & ${ }^{\mathbf{c}} \mathrm{BCR}$ \\
\hline \multirow{3}{*}{ 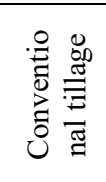 } & $\begin{array}{l}\text { Cropping } \\
\text { system }\end{array}$ & $\begin{array}{l}\text { Wheat Yield } \\
\quad\left(\mathrm{t} \mathrm{ha}^{-1}\right)\end{array}$ & $\begin{array}{l}\text { Wheat Yield } \\
\text { Value } \\
\text { (US\$. ha-1) }\end{array}$ & $\begin{array}{l}\text { Straw yield } \\
\left(\mathrm{t} \mathrm{ha}^{-1}\right)\end{array}$ & $\begin{array}{l}\text { Straw Value } \\
\left(\mathrm{US} \$ . \mathrm{ha}^{-1}\right)\end{array}$ & $\begin{array}{l}{ }^{\mathrm{a}} \text { Gross } \\
\text { income } \\
\left(\mathrm{US} \$ \mathrm{ha}^{-1}\right)\end{array}$ & $\begin{array}{l}\text { Total cost } \\
\left(\mathrm{US} \$ \mathrm{ha}^{-1}\right)\end{array}$ & $\begin{array}{l}{ }^{\mathrm{b}} \text { Net field } \\
\text { benefit } \\
\text { (US\$. } \text { ha }^{-1} \text { ) }\end{array}$ & ${ }^{\mathrm{c}} \mathrm{BCR}$ \\
\hline & Cotton-wheat & $3.67 \pm 0.01^{\mathrm{cd}}$ & $819.20 \pm 3.18^{\mathrm{cd}}$ & $10.23 \pm 0.04^{b}$ & $116.56 \pm 0.46^{\mathrm{b}}$ & $935.76 \pm 3.53^{\mathrm{cd}}$ & $413.85 \pm 3.10^{\mathrm{ab}}$ & $521.91 \pm 5.21^{\mathrm{cd}}$ & $2.28 \pm 0.15^{\mathrm{de}}$ \\
\hline & Maize-wheat & $3.76 \pm 0.04^{\mathrm{c}}$ & $838.81 \pm 3.80^{\mathrm{c}}$ & $10.40 \pm 0.05^{\mathrm{ab}}$ & $118.48 \pm 0.60^{\mathrm{ab}}$ & $957.29 \pm 4.25^{\mathrm{c}}$ & $415.81 \pm 5.08^{\mathrm{a}}$ & $541.47 \pm 4.08^{\mathrm{c}}$ & $2.32 \pm 0.13^{\mathrm{cd}}$ \\
\hline \multirow{3}{*}{ 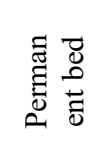 } & $\begin{array}{l}\text { Mungbean- } \\
\text { wheat }\end{array}$ & $3.55 \pm 0.05^{\mathrm{d}}$ & $791.20 \pm 4.09^{\mathrm{d}}$ & $10.10 \pm 0.04^{b}$ & $115.11 \pm 0.42^{b}$ & $906.31 \pm 4.91^{\mathrm{d}}$ & $411.05 \pm 4.09^{b}$ & $495.26 \pm 4.38^{\mathrm{d}}$ & $2.22 \pm 0.13^{\mathrm{e}}$ \\
\hline & Cotton-wheat & $4.08 \pm 0.01^{\mathrm{a}}$ & $909.50 \pm 2.04^{\mathrm{a}}$ & $10.64 \pm 0.04^{\mathrm{a}}$ & $121.21 \pm 0.44^{\mathrm{a}}$ & $1030.71 \pm 2.45^{\mathrm{a}}$ & $372.85 \pm 0.24^{\mathrm{c}}$ & $657.86 \pm 2.24^{\mathrm{a}}$ & $2.76 \pm 0.01^{\mathrm{a}}$ \\
\hline & Maize-wheat & $4.11 \pm 0.02^{\mathrm{a}}$ & $916.48 \pm 4.86^{\mathrm{a}}$ & $10.64 \pm 0.04^{\mathrm{a}}$ & $121.18 \pm 0.45^{\mathrm{a}}$ & $1037.66 \pm 5.31^{\mathrm{a}}$ & $373.55 \pm 0.49^{c}$ & $664.11 \pm 4.82^{\mathrm{a}}$ & $2.78 \pm 0.01^{\mathrm{a}}$ \\
\hline \multirow{4}{*}{$\stackrel{\circ}{\stackrel{0}{\Xi}}$} & $\begin{array}{l}\text { Mungbean- } \\
\text { wheat }\end{array}$ & $3.90 \pm 0.02^{\mathrm{b}}$ & $870.82 \pm 4.19^{b}$ & $10.67 \pm 0.18^{\mathrm{a}}$ & $121.54 \pm 2.02^{\mathrm{a}}$ & $992.36 \pm 2.36^{b}$ & $368.98 \pm 0.42^{\mathrm{d}}$ & $623.38 \pm 1.91^{\mathrm{b}}$ & $2.69 \pm 0.01^{\mathrm{b}}$ \\
\hline & Cotton-wheat & $2.84 \pm 0.03^{\mathrm{e}}$ & $632.93 \pm 5.75^{\mathrm{e}}$ & $8.31 \pm 0.01^{\mathrm{c}}$ & $94.73 \pm 0.14^{\mathrm{c}}$ & $727.66 \pm 5.71^{\mathrm{e}}$ & $315.88 \pm 0.57^{\mathrm{e}}$ & $411.79 \pm 5.22^{\mathrm{e}}$ & $2.30 \pm 0.01^{\mathrm{cd}}$ \\
\hline & Maize-wheat & $2.92 \pm 0.04^{\mathrm{e}}$ & $651.67 \pm 4.89^{\mathrm{e}}$ & $8.24 \pm 0.04^{\mathrm{c}}$ & $93.92 \pm 0.49^{\mathrm{c}}$ & $745.59 \pm 4.32^{\mathrm{e}}$ & $317.75 \pm 0.89^{\mathrm{e}}$ & $427.84 \pm 8.43^{\mathrm{e}}$ & $2.35 \pm 0.02^{\mathrm{c}}$ \\
\hline & $\begin{array}{l}\text { Mungbean- } \\
\text { wheat }\end{array}$ & $2.91 \pm 0.03^{\mathrm{e}}$ & $650.09 \pm 5.45^{\mathrm{e}}$ & $8.42 \pm 0.06^{\mathrm{c}}$ & $95.98 \pm 0.65^{\mathrm{c}}$ & $746.06 \pm 6.08^{\mathrm{e}}$ & $317.59 \pm 0.55^{\mathrm{e}}$ & $428.47 \pm 5.54^{\mathrm{e}}$ & $2.35 \pm 0.02^{\mathrm{c}}$ \\
\hline
\end{tabular}

${ }^{a}$ Gross income were calculated by multiplying the field price of crop by the adjusted yield; field price of crop was calculated by taking the price that farmers receive for crop when

they sell it, and subtracting all the associated costs associated with harvest, and sale proportional to the yield.

${ }^{\mathrm{b}}$ Net field benefits are calculated by subtracting gross income from total costs.

${ }^{c} \mathrm{BCR}$; benefit cost ratio 
Table 5a. Effect of different tillage system on marginal analysis of Kharif crops.

\begin{tabular}{|c|c|c|c|c|c|c|}
\hline Crop & Tillage & $\begin{array}{l}\text { Cost that vary } \\
\left.\text { (US\$. } \text { ha }^{-1}\right)\end{array}$ & $\begin{array}{l}\text { Marginal cost that vary } \\
\left(\mathrm{USS} . \mathrm{ha}^{-1}\right)\end{array}$ & $\begin{array}{c}\text { Net field benefit } \\
\left(\text { US\$. ha }{ }^{-1}\right)\end{array}$ & $\begin{array}{c}\text { Marginal net benefits } \\
\left.\text { (US\$. } \text { ha }^{-1}\right)\end{array}$ & $\begin{array}{c}\text { Marginal rate of return } \\
(\%)\end{array}$ \\
\hline \multirow{3}{*}{ Cotton } & No tillage & 139.26 & - & 387.26 & - & \\
\hline & Permanent beds & 186.13 & 46.87 & 948.67 & 561.42 & 1198 \\
\hline & Traditional tillage & 276.68 & 90.56 & 674.47 & -274.21 & -303 \\
\hline \multirow{3}{*}{ Maize } & No tillage & 108.97 & - & 193.05 & - & - \\
\hline & Permanent beds & 125.69 & 1761 & 425.40 & 232.35 & 1389 \\
\hline & Traditional tillage & 209.36 & 83.67 & 401.52 & -23.89 & -29 \\
\hline & No tillage & 100.99 & - & 457.95 & - & - \\
\hline \multirow{2}{*}{ Mungbean } & Permanent beds & 135.48 & 34.49 & 788.34 & 330.39 & 958 \\
\hline & Traditional tillage & 167.27 & 31.79 & 689.81 & -98.53 & -310 \\
\hline
\end{tabular}

Table 5b. Effect of different tillage system and crop rotation on marginal analysis of wheat.

\begin{tabular}{|c|c|c|c|c|c|c|}
\hline Tillage & Cropping system & $\begin{array}{l}\text { Cost that vary } \\
(\text { US\$. ha }\end{array}$ & $\begin{array}{l}\text { Marginal cost } \\
\left.(\text { US\$. ha })^{-1}\right)\end{array}$ & $\begin{array}{c}\text { Net field benefits } \\
\left(\text { US\$. } \mathrm{ha}^{-1}\right)\end{array}$ & $\begin{array}{l}\text { Marginal net } \\
\left(\text { US\$. ha }^{-1}\right)\end{array}$ & Marginal rate (\%) \\
\hline \multirow{3}{*}{ Conventional tillage } & Cotton-wheat & 111.01 & - & 411.78 & - & - \\
\hline & Maize-wheat & 167.99 & 56.97 & 657.86 & 246.07 & 432 \\
\hline & Mungbean-wheat & 208.99 & 41.00 & 521.91 & -135.95 & -332 \\
\hline \multirow{3}{*}{ Permanent bed } & Cotton-wheat & 112.89 & - & 427.84 & - & - \\
\hline & Maize-wheat & 168.68 & 55.79 & 664.11 & 236.28 & 423 \\
\hline & Mungbean-wheat & 210.96 & 42.26 & 541.47 & -122.64 & -290 \\
\hline \multirow{3}{*}{ No tillage } & Cotton-wheat & 112.73 & - & 428.48 & - & - \\
\hline & Maize-wheat & 164.12 & 51.39 & 623.38 & 194.91 & 379 \\
\hline & Mungbean-wheat & 206.19 & 42.07 & 495.26 & -128.13 & -305 \\
\hline
\end{tabular}

Table 6. Economic yield of individual crop, wheat equivalent yield (WEY), Production efficiency and Land resource use efficiency (LRUE) of different Wheat based cropping systems (pooled data for cropping seasons).

\begin{tabular}{|c|c|c|c|c|c|c|c|}
\hline Tillage system & Econ & $\begin{array}{l}\text { yield } \\
1)\end{array}$ & $\begin{array}{c}{ }^{\mathrm{a}} \text { WEY kharif } \\
\left(\mathrm{t} \mathrm{ha}^{-1}\right)\end{array}$ & $\begin{array}{c}{ }^{\mathrm{b}} \text { WEY system } \\
\left(\mathrm{t} \mathrm{ha}^{-1}\right)\end{array}$ & $\begin{array}{c}\text { Total duration } \\
\text { (days) }\end{array}$ & $\begin{array}{c}\text { Production efficiency } \\
\text { (kg/ha/day) }\end{array}$ & $\begin{array}{c}\text { LRUE } \\
(\%)\end{array}$ \\
\hline & Cotton & Wheat & & & & & \\
\hline Conventional tillage & $2.91 \pm 0.03^{\mathrm{b}}$ & $3.67 \pm 0.01^{\mathrm{cd}}$ & $5.59 \pm 0.03^{\mathrm{b}}$ & $9.26 \pm 0.04^{\mathrm{b}}$ & $167+149=318$ & $17.57 \pm 0.08^{b}$ & 87.12 \\
\hline Permanent bed & $3.21 \pm 0.02^{\mathrm{a}}$ & $4.08 \pm 0.04^{\mathrm{c}}$ & $6.18 \pm 0.02^{\mathrm{a}}$ & $10.26 \pm 0.04^{\mathrm{a}}$ & $167+149=318$ & $23.07 \pm 0.09^{\mathrm{a}}$ & 87.12 \\
\hline No tillage & $\begin{array}{c}2.19 \pm 0.05^{\mathrm{c}} \\
\mathrm{M}^{\mathrm{ai}} \mathrm{z}^{\mathrm{e}}\end{array}$ & $\begin{array}{l}2.84 \pm 0.05^{\mathrm{d}} \\
\mathrm{W}^{\text {hea }} \mathrm{t}\end{array}$ & $4.21 \pm 0.05^{\mathrm{c}}$ & $7.05 \pm 0.06^{\mathrm{c}}$ & $167+149=318$ & $13.24 \pm 0.12^{\mathrm{c}}$ & 87.12 \\
\hline Conventional tillage & $6.38 \pm 0.04^{\mathrm{a}}$ & $3.76 \pm 0.01^{\mathrm{a}}$ & $4.42 \pm 0.04^{\mathrm{a}}$ & $8.18 \pm 0.05^{\mathrm{b}}$ & $119+149=268$ & $16.48 \pm 0.05^{\mathrm{a}}$ & 73.42 \\
\hline Permanent bed & $6.12 \pm 0.04^{\mathrm{b}}$ & $4.11 \pm 0.02^{\mathrm{a}}$ & $4.24 \pm 0.04^{\mathrm{b}}$ & $8.34 \pm 0.01^{\mathrm{a}}$ & $119+149=268$ & $15.81 \pm 0.01^{\mathrm{b}}$ & 73.42 \\
\hline No tillage & $\begin{array}{l}4.96 \pm 0.02^{\mathrm{c}} \\
\mathrm{Mun}^{\mathrm{g}}\end{array}$ & $\begin{array}{c}2.92 \pm 0.02^{\mathrm{b}} \\
\mathrm{W}^{\text {hea }} \mathrm{t}\end{array}$ & $3.44 \pm 0.02^{\mathrm{c}}$ & $6.36 \pm 0.04^{c}$ & $119+149=268$ & $12.82 \pm 0.12^{\mathrm{c}}$ & 73.42 \\
\hline Conventional tillage & $1.35 \pm 0.01^{\mathrm{b}}$ & $3.55 \pm 0.03^{\mathrm{e}}$ & $5.19 \pm 0.01^{\mathrm{b}}$ & $8.74 \pm 0.06^{\mathrm{b}}$ & $110+149=259$ & $20.06 \pm 0.05^{\mathrm{a}}$ & 70.96 \\
\hline Permanent bed & $1.43 \pm 0.02^{\mathrm{a}}$ & $3.90 \pm 0.04^{\mathrm{e}}$ & $5.49 \pm 0.02^{\mathrm{a}}$ & $9.40 \pm 0.02^{\mathrm{a}}$ & $110+149=259$ & $17.28 \pm 0.05^{\mathrm{b}}$ & 70.96 \\
\hline No tillage & $1.00 \pm 0.02^{\mathrm{c}}$ & $2.91 \pm 0.03^{\mathrm{e}}$ & $3.85 \pm 0.02^{\mathrm{c}}$ & $6.77 \pm 0.04^{\mathrm{c}}$ & $110+149=259$ & $14.38 \pm 0.3^{\mathrm{c}}$ & 70.96 \\
\hline
\end{tabular}


Conclusion: In conclusion of present study, the result counseled that cotton-wheat was the ultimate best crop sequence in mixed-cropping in terms of economic gains. The cotton-wheat cropping system gave highest net field benefit (NFB) and marginal rate of return (MRR) in the semiarid environment. The cotton-wheat cultivation in permanent beds gave highest land use efficiency. Moreover, highest production efficiency was also ascertained in cotton-wheat cultivation system in permanent bed. Thus, present study helps the farmer to choose cropping system according to the availability of resources.

\section{REFERENCES}

Akbarnia, A., and F. Farhani (2014). Study of fuel consumption in three tillage methods. Res. Agric. Eng. 60: 142-147.

Bari, M.N., and M.R. Islam (2009). Selection of mungbean variety for rice-wheat-fallow cropping system-a participatory research and development (PR\&D) approach. J. Agri. Rural. Dev. 7: 33-40.

Bertolino, A. V. N. F. Fernandes, J. P. Miranda, A. P. Souza, M. R. Lopes and F. Palmieri (2010). Effects of plough pan development on surface hydrology and on soil physical properties in Southeastern Brazilian plateau. J. Hydrology, 393: 94-104.

Boulal, H., H. Gomez-Macphersona, and F.J. Villalobosa (2012). Permanent bed planting in irrigated Mediterranean conditions: short-term effects on soil quality, crop yield and water use efficiency. Field Crops Res. 130: 120-127.

Braun, H.J., G. Atlin, and T. Payne (2010). Multilocation testing as a tool to identify plant response to global climate change. In: M.P. Reynolds (ed.), Climate change and crop production. CABI, London.

Cimmyt, (1988). From agronomic data to farmer recommendations: an economics training manual. Mexico, D.F, 1988: pp79.

Connor, D.J., J. Timsina, and E. Humphreys (2003). Prospects for permanent beds for the rice-wheat system. In: Improving the Productivity and Sustainability of Rice-Wheat Systems: Issue and Impact. ASA Special Publication 65: 197210.

Crookston, R.K. (1995). The rotation effect in corn: A summary of fifteen years of field research in Minnesota. Minnesota Agric. Univ. of Minnesota, Exp. Stn. 22-180.

David T., K.G. Cassman, P.A. Matson, R. Naylor and S. Polasky (2002). Agricultural sustainability and intensive production practices. Nat. 418: 671677.
Dawe, D. (2008). Have recent increases in international cereal prices been transmitted to domestic economies. The experience in seven large Asian countries. FAO-ESA Working Paper, 8: 1-12.

De Vita, P., E. Di Paolo, G. Fecondo, N. Di Fonzo, and M. Pisante (2007). No-tillage and conventional tillage effects on durum wheat yield, grain quality and soil moisture content in southern Italy. Soil Till. Res. 92: 69-78.

Erenstein, O., and V. Laxmi (2008). No tillage impacts in India's rice-wheat systems. Soil Till. Res. 100: $1-14$.

FAO (2014). FAO statistical databases FAOSTAT. http://faostat3.fao.org/ Accessed 8 Jun 2014.

Farooq, M., K. C., Flower, K., Jabran, A., Wahid, and K. H. Siddique (2011). Crop yield and weed management in rainfed conservation agriculture. Soil Till. Res. 117: 172-183.

Peter, S. (2011). Foresight: the future of food and farming, final project report, Prometheus, 29: 309-313.

Friedrich, T., R. Derpsch, and A. Kassam (2017). Overview of the global spread of conservation agriculture. In Sustainable Development of Organic Agriculture pp. 75-90

Garcua-Torres, L. (2000). Conservation tillage in Europe: a needed challenge. In: CIMMYT and ICARDA. Conservation tillage: A viable option for sustainable agriculture in Central Asia. Almaty, Kazakhstan: Aleppo, Syria: ICARDA.

Gathala, M.K., J.K. Ladha, Y.S. Saharawat, V. Kumar, V. Kumar, P.K. Sharma (2011). Effect of tillage and crop establishment methods on physical properties of a medium-textured soil under a seven-year rice-wheat rotation. Soil Sci. Soc. Am. J. 75: 1851-1862.

Higgs, R.L., A.E. Peterson and W.H. Paulson (1990). Crop rotations: Sustainable and profitable. J. Soil Water Conserv. 45: 68-70.

Hobbs, PR. (2007). Conservation agriculture: what is it and why is it important for future sustainable food production?. The J. Agric. Sci. 1: 145:127.

Iqbal, M., M.A. Khan and M.Z. Anwar (2002). Zerotillage technology and farm profits: A case study of wheat growers in rice zone of Punjab. Pakistan Dev. Review 41: 665-682.

Khalid, U., N. Khan, M.U. Khan, F. Y. Saleem and A. Rashid (2014). Impact of tillage and nitrogen on cotton yield and quality in a wheat-cotton system, Pakistan. Archives of Agron. and Soil Sci. 4: 519-530.

Khan, M.B. and A. Khaliq (2005). Production of winter cereals as relay crops by surface seeding in cotton based cropping system. J. Res. Sci. 16: 79-86. 
Khan, M.B., R. Rafiq, M. Hussain, M. Farooq, K. Jabran (2012). Ridge sowing improves root system, phosphorus uptake, growth and yield of maize (Zea mays L.) hybrids. The J. Anim. Plant Sci. 22: 309-317.

Kienzler, K.M., J.P.A. Lamers, A. McDonald, A. Mirzabaev, N. Ibragimov, O. Egamberdiev, E. Ruzibaev, and A. Akramkhanov (2012). Conservation agriculture in Central Asia What do we know and where do we go from here? Field Crops Res. 132: 95-105.

Kundu, D., and B. Mahapatra (2014). System productivity, profitability and resource use efficiency of jute (Corchorus olitorius) based cropping systems in the eastern Indo-gangetic plain. Ind. J. Agric. Sci. 84: 209-13.

Ladha, J.K., D. Dawe, H. Pathak, A.T. Padre, R.L. Yadav, B. Singh, Y. Singh, Y. Singh, P. Singh, A.L. Kundu, R. Sakal, N. Ram, A.P. Regmi, S.K. Gami, Bhandari, A.L. Amin, C.R. Yadav, E.M. Bhattarai, S. Das, H.P. Aggarwal, R.K. Gupta, and P.R. Hobbs (2003). How extensive are yield declines in long-term rice- wheat experiments in Asia. Field Crops Res. 81: 159180.

Micucci, F. G., and M. A. Taboada (2006). Soil physical properties and soybean (Glycine max, Merrill) root abundance in conventionally-and zero-tilled soils in the humid Pampas of Argentina. Soil Till. Res. 86(2): 152-162.

Naresh, R.K., S.P. Singh and P. Chauhan (2012). Influence of conservation agriculture, permanent raised bed planting and residue management on soil quality and productivity in maize-wheat system in Western Utter Pradesh. Int. J. Life Sc. Bt. Pharm. Res. 1: 27-33.

Ratnadass, A., P. Fernandes J. Avelino and R. Habib (2012). Plant species diversity for sustainable management of crop pests and diseases in agroecosystems: a review. Agr. Sus. Dev. 32(1): 273-303.

Sainju, U.M., Z.N. Senwo, E.Z. Nyakatawa I.A. Tazisong and K.C. Reddy (2008). Tillage, cropping systems, and nitrogen fertilizer source effects on soil carbon sequestration and fractions. J. Environ. Qual. 37: 880-888.
Schlegel, A.J., Y. Assefa, T.J., Dumler, L.A., Haag, L.R., Stone, A.D., Halvorson, and C.R., Thompson (2016). Limited irrigation of cornbased no-till crop rotations in west central Great Plains. Agron. J. 108: 1132-1141.

Shah, M.A., M. Farooq and M. Hussain (2016). Productivity and profitability of cotton-wheat system as influenced by relay intercropping of insect resistant transgenic cotton in bed planted wheat. Euro. J. Agr. 75: 33-41.

Shahzad, M., M. Farooq and M. Hussain (2016). Weed spectrum in different wheat-based cropping systems under conservation and conventional tillage practices in Punjab, Pakistan. Soil Till. Res. 163: 71-79.

Shahzad, M., M. Hussain, M. Farooq, S. Farooq, K. Jabran and A. Nawaz (2017). Economic assessment of conventional and conservation tillage practices in different wheat-based cropping systems of Punjab, Pakistan. Environ. Sci. Poll. Res. 24: 24634-24643.

Tanaka, D. L., J.M. Krupinsky, M.A. Liebig, S.D. Merrill, R.E. Ries, J.R. Hendrickson, H.A. Johnson and J. D. Hanson (2002). Dynamic cropping systems. Agr. J. 5: 957- 961

Tilman, D., K.G. Cassman, P.A. Matson, R. Naylor and S. Polasky (2002). Agricultural sustainability and intensive production practices. Nat. 418: 671.

Timsina, J., and D. J. Connor (2001). Productivity and management of rice-wheat cropping systems: issues and challenges. Field Crop Res. 69: 93132.

USDA, N. 2016. The PLANTS Database (http://plants. usda. gov). National plant data team, Greensboro, NC 27401-4901 USA. Accessed29 January.

Zandastra, H. G. (1976). Cropping system research for the Asian farmers. In Proceedings of the Symposium on Cropping Systems Research and Development for the Asian farmer, IRRI, Manila, pp. 21-24.

Zhang, W., L.B., Hou, B., Zhang, J., Wen, G.J., Wang, W.C., Jiang, Y., Jia, 2006. Effects of different cultivation ways on soil physical capability in western semi-arid area of Liaoning Province. J. Arid Land Resour. Environ. 3: 149-153. 